\title{
Morule-like features in pulmonary adenocarcinoma associated with epidermal growth factor receptor mutations: two case reports with targeted next-generation sequencing analysis
}

\author{
Yoo Jin Lee, Harim Oh, Eojin Kim, Bokyung Ahn, Jeong Hyeon Lee, Youngseok Lee, Yang Seok Chae, Chul Hwan Kim \\ Department of Pathology, Korea University Anam Hospital, Seoul, Korea
}

\begin{abstract}
Morules, or morule-like features, can be identified in benign and malignant lesions in various organs. Morular features are unusual in pulmonary adenocarcinoma cases with only 26 cases reported to date. Here, we describe two cases of pulmonary adenocarcinoma with morule-like features in Korean women. One patient had a non-mucinous-type adenocarcinoma in situ and the other had an acinarpredominant adenocarcinoma with a micropapillary component. Both patients showed multiple intra-alveolar, nodular, whorled proliferative foci composed of atypical spindle cells with eosinophilic cytoplasm. Targeted next-generation sequencing was performed on DNA extracted from formalin-fixed paraffin-embedded samples of the tumors. Results showed unusual epidermal growth factor receptor (EGFR) mutations, which are associated with drug resistance to EGFR tyrosine kinase inhibitors, revealing the importance of identifying morule-like features in pulmonary adenocarcinoma and the need for additional study, since there are few reported cases.
\end{abstract}

Key Words: Morule-like features; Pulmonary adenocarcinoma; Next-generation sequencing; Epidermal growth factor receptor

Received: April 30, 2019 Revised: August 28, 2019 Accepted: September 26, 2019

Corresponding Author: Chul Hwan Kim, MD, PhD, Department of Pathology, Korea University Anam Hospital, 73 Inchon-ro, Seongbuk-gu, Seoul 02841, Korea

Tel: +82-2-920-5595, Fax: +82-2-927-6576, E-mail: chkap@korea.ac.kr

Morules, or morule-like features, can be identified in both benign and malignant lesions in various organs [1]. A subset of pulmonary adenocarcinomas show morule-like features, particularly papillary-predominant adenocarcinoma [2]. Morules can also be identified in adenocarcinoma in situ (AIS); previously known as bronchioloalveolar carcinoma [3]. One case in this report showed morule-like features in AIS and the other case showed these features in invasive adenocarcinoma. Using next-generation sequencing (NGS), we attempted to identify the molecular characteristics of these rare morular features in the two cases.

\section{CASE REPORT}

The Institutional Review Board of Korea University Anam Hospital (2018AN0182) approved this study, and we received informed consent from both patients.

\section{Case 1}

A healthy, 78-year-old Korean woman was admitted to the hospital with an incidental mass in the left lung. The computed tomography (CT) scan of the left upper lung showed a sub-solid nodule measuring $23 \mathrm{~mm}$ in diameter, suggestive of AIS or minimally invasive adenocarcinoma. The patient had no surgical history, had never smoked, and was relatively healthy. A segmentectomy was performed to remove the pulmonary mass. Grossly, the resected lung measured $10.3 \times 5.3 \times 4.5 \mathrm{~cm}$ and weighed $30 \mathrm{~g}$ in total. The pleural surface of the mass was smooth. The tumor measured $2.2 \times 1.8 \mathrm{~cm}$, was fibrotic and whitish and grey colored without hemorrhage or necrosis, and did not involve the pleura. Hematoxylin and eosin-stained histology sections showed lepidic growth of atypical columnar cells with enlarged nuclei and irregular nuclear contours (Fig. 1A). There were multiple intraalveolar nodular proliferative foci composed of atypical spindle cells with eosinophilic cytoplasm (Fig. 1B). Cells in the nodular proliferative lesions were arranged in a whorled pattern, normally interpreted with a squamoid appearance, yet obvious squamous differentiation was not seen. The underlying pulmonary architecture was preserved, which suggested that stromal invasive foci 

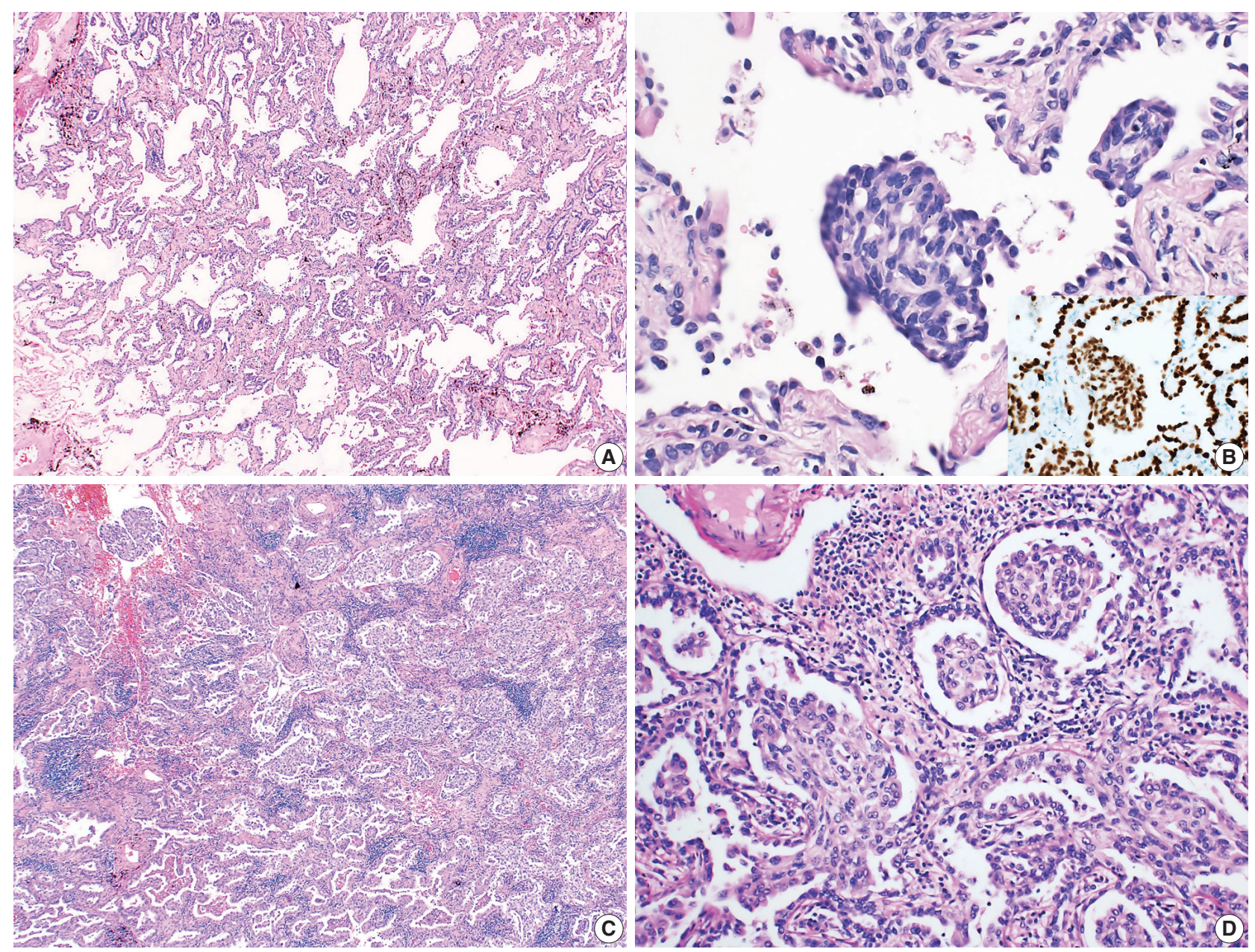

Fig. 1. Microscopic findings of the tumor. (A) The case of adenocarcinoma in situ shows lepidic growth of atypical columnar cells without definite evidence of stromal invasion. (B) Multiple nodular proliferative lesions in intra-alveolar spaces. The cells in those structures are atypical spindle cells that are positive for thyroid transcription factor 1 immunostaining (inset). (C) The acinar-predominant adenocarcinoma case shows multiple foci of morule-like components (D) floating within the intra-alveolar space.

were not present. Neither lymphovascular nor pleural invasion was evident; therefore, the final diagnosis was non-mucinous type AIS. Upon immunohistochemistry, the cells forming morule-like features were positive for thyroid transcription factor 1 (TTF-1) and cytokeratin (CK), but negative for p40, CDX-2, neuron-specific enolase, chromogranin, and synaptophysin (Fig. $1 \mathrm{~B}$, inset). There were no remarkable features in normal lung tissue. We also performed NGS analysis and revealed an insertion in epidermal growth factor receptor (EGFR) exon 20 (NM_001346897.1: c.2180_2185dup) with 35.5\% mutation abundance. There was no evidence of recurrence or metastasis at 1-year follow-up.

\section{Case 2}

A healthy, 46-year-old Korean woman was admitted to the hospital with an incidental mass in one lung. The CT scan of the right lower lung showed a sub-solid nodule measuring 29 $\mathrm{mm}$ in diameter with a 12-mm-sized solid portion. The mass was associated with apical pleural thickening, suggestive of a malignant tumor with pleural invasion. The patient had no surgical history, had never smoked, and was relatively healthy. A lobectomy was performed to remove the sub-solid nodule from the right lower lung. Grossly, the resected lung specimen was $16.9 \times 14.5 \times 4.3 \mathrm{~cm}$. The pleural surface showed umbilicated foci, suggestive of pleural invasion. Upon sectioning, the cut surface showed an ill-demarcated, whitish-grey, fibrotic mass measuring $2.7 \times 1.3 \mathrm{~cm}$ with pleural invasion. Upon microscopic examination, the tumor was identified as an acinar-predominant adenocarcinoma with a micropapillary component. In the region of the acinar-predominant adenocarcinoma, multiple 
morule-like foci were identified floating within the intra-alveolar space without clear evidence of squamous differentiation (Fig. $1 C, \mathrm{D})$. In accordance with the gross findings, visceral pleural invasion was confirmed by microscopy. Lymphatic invasion was also evident, yet there was no lymph node metastasis. Additional studies were performed to determine molecular characteristics of the morule-like component. Fluorescent in situ hybridization for anaplastic lymphoma kinase $(A L K)$ gene rearrangement was performed on a preoperative diagnostic needle biopsy specimen and showed split signals in four out of 100 analyzed cancer cells, indicating the absence of $A L K$ rearrangement. We performed NGS analysis on a formalin-fixed paraffin-embedded section of a surgical specimen, and a $\mathrm{L} 747 \mathrm{~S}$ missense mutation was detected in EGFR exon 19 (NM_005228.3: c.2240T > C) with $24.8 \%$ mutation abundance. There was no recurrence or metastasis at 1-year follow-up.

\section{DISCUSSION}

Adenocarcinoma with morule-like features is a rare variant of lung adenocarcinoma, accounting for $1.9 \%$ of all adenocarcinomas [2]. To date, 26 cases of lung adenocarcinoma with morulelike features have been reported [1-5] that were reported as papillary (15 cases), micropapillary (3 cases), acinar (3 cases), and solid-predominant adenocarcinoma (3 cases). The remaining two cases were adenocarcinoma with a lepidic pattern [2], and bronchioloalveolar carcinoma (AIS) [3].

Histologically, the cells comprising the morular feature were spindle shaped and distinct from the other cancer cells. There are several possibilities to explain the pathophysiology of this unusual morular component. Traditionally, morules have been reported in pulmonary blastoma and well-differentiated fetal adenocarcinoma, and are regarded as non-epithelial cell clusters showing neuronal differentiation $[1,6]$. However, morular cells in the cases reported here were positive for pulmonary epithelial markers, including CK and TTF-1, but negative for neuroendocrine markers, consistent with several other reports [1-3,5]. As such, they can be considered a complex glandular component, or epithelial cell nodules. Therefore, the presence of a morular component in small biopsy specimens does not, by itself, indicate pulmonary blastoma and immunohistochemistry can help to determine the likelihood of epithelial carcinoma. Furthermore, although the morular cells are spindle shaped, Moran et al. [5] reported that negative results for muscle markers and human melanoma black-45 reduce the probability of smooth muscle tumor and lymphangioleiomyomatosis.
Fornelli et al. [3] reported the first case of AIS with morulelike features as a non-invasive tumor because both architectural complexity and stromal desmoplasia were not apparent. Fornelli's case is the only report currently available in the literature, making our reported cases the second study of AIS showing morule-like features. Tsuta et al. [2] found that lung adenocarcinomas with morule-like features have lower 5-year overall survival rates, suggesting that morule-like features are an invasive component similar to an aggressive histologic micropapillary pattern. On the other hand, one report suggested that the morular feature itself might not have prognostic significance [7], and the invasion criteria for the pulmonary adenocarcinoma used in World Health Organization 2015 classification of tumors of the lung, pleura, thymus, and heart does not include morular features. Therefore, if there is no other evidence of stromal invasion, AIS with morular features should be evaluated separately from the invasive adenocarcinoma. This case of AIS did not show any evidence of recurrence or metastasis at 1-year followup; however, further studies of AIS with morule-like features are needed.

Depending on the observer, morular like components filling the intra-alveolar space can be considered as an invasive lesion, or an airspace invasion forming a solid nest. From that point of view, case 1 described here can be diagnosed as lepidic predominant adenocarcinoma, as morular portion size is $0.7 \mathrm{~cm}$ in maximum diameter. However, further research will be needed as some argue that there is no prognostic significance [7].

The molecular characteristics of the morular component are not fully understood. However, several studies have revealed that this feature is associated with EGFR mutations. In a previous report that analyzed two common EGFR mutations, including deletions in exon 19 and a point mutation at codon 858 in exon 21 (L858R), the presence of morule-like components correlated with EGFR mutations, particularly deletions, and was reported to be an independent predictive factor of EGFR mutation status [2]. Another report by Tajima and Koda [4] found that morulelike features were associated with a deletion mutation at exon 19 in EGFR. Ours is the first report in which NGS was used to identify the molecular characteristics of morule-like features in pulmonary adenocarcinoma. Our results revealed unusual EGFR mutations that have not been previously reported in pulmonary adenocarcinoma with morule-like features. The rare mutation in EGFR exon 19 and the insertion mutation in EGFR exon 20 can be associated with drug resistance to EGFR tyrosine kinase inhibitors [8-10], implying the importance of identifying morulelike features in pulmonary adenocarcinoma. 
Morule-like components are a rare histologic feature of pulmonary adenocarcinoma, which may be associated with rare EGFR mutations, such as the $\mathrm{L} 747 \mathrm{~S}$ missense mutation of exon 19 or an insertion mutation in exon 20. The presence of morulelike features without definite invasive components led to the diagnosis of AIS in one of these cases, as it is not yet clear whether this feature needs to be considered as an invasive component. Additional studies are needed to clarify the significance of this rare histologic variant in pulmonary adenocarcinoma.

\section{ORCID}

Yoo Jin Lee: https://orcid.org/0000-0003-3830-7051

Harim Oh: https://orcid.org/0000-0003-4904-4015

Eojin Kim: https://orcid.org/0000-0003-3111-6754

Bokyung Ahn: https://orcid.org/0000-0002-0229-2276

Jeong Hyeon Lee: https://orcid.org/0000-0003-2041-4617

Youngseok Lee: https://orcid.org/0000-0002-9762-4957

Yang Seok Chae: https://orcid.org/0000-0002-8801-0910

Chul Hwan Kim: https://orcid.org/0000-0003-2026-8824

\section{Author Contributions}

Conceptualization: YJL, CHK.

Data curation: EK, BA.

Investigation: $\mathrm{YJL}, \mathrm{HO}$.

Project administration: YJL, JHL.

Resources: YL, YSC.

Supervision: YSC, CHK.

Validation: YL, YSC.

Visualization: YJL, JHL.

Writing - original draft: YJL.

Writing - review \& editing: CHK.

\section{Conflicts of Interest}

The authors declare that they have no potential conflicts of interest.

\section{Funding}

No funding to declare.

\section{REFERENCES}

1. Makishi S, Kinjo T, Sawada S, et al. Morules and morule-like features associated with carcinomas in various organs: report with immunohistochemical and molecular studies. J Clin Pathol 2006; 59: 95-100.

2. Tsuta K, Kawago M, Yoshida A, et al. Primary lung adenocarcinoma with morule-like components: a unique histologic hallmark of aggressive behavior and EGFR mutation. Lung Cancer 2014; 85: 12-8.

3. Fornelli A, Cavazza A, Cancellieri A, Rossi G, De Marco L. Bronchioloalveolar carcinoma with nodular ("morule-like") features. Virchows Arch 2003; 442: 407-8.

4. Tajima S, Koda K. Transition between morule-like and solid components may occur in solid-predominant adenocarcinoma of the lung: report of 2 cases with EGFR and KRAS mutations. Int J Clin Exp Pathol 2015; 8: 7475-81.

5. Moran CA, Jagirdar J, Suster S. Papillary lung carcinoma with prominent "morular" component. Am J Clin Pathol 2004; 122: 106-9.

6. Nakatani Y, Masudo K, Miyagi Y, et al. Aberrant nuclear localization and gene mutation of beta-catenin in low-grade adenocarcinoma of fetal lung type: up-regulation of the Wnt signaling pathway may be a common denominator for the development of tumors that form morules. Mod Pathol 2002; 15: 617-24.

7. Matsukuma S, Obara K, Kato K, et al. Non-sarcomatous spindle cell morphology in conventional lung adenocarcinoma: a clinicopathological study. Virchows Arch 2014; 465: 165-72.

8. Suda K, Mizuuchi H, Maehara Y, Mitsudomi T. Acquired resistance mechanisms to tyrosine kinase inhibitors in lung cancer with activating epidermal growth factor receptor mutation: diversity, ductility, and destiny. Cancer Metastasis Rev 2012; 31: 807-14.

9. Yamaguchi F, Fukuchi K, Yamazaki Y, et al. Acquired resistance L747S mutation in an epidermal growth factor receptor-tyrosine kinase inhibitor-naive patient: a report of three cases. Oncol Lett 2014; 7: 357-60.

10. Yasuda H, Kobayashi S, Costa DB. EGFR exon 20 insertion mutations in non-small-cell lung cancer: preclinical data and clinical implications. Lancet Oncol 2012; 13: e23-31. 